\title{
Detection and measurement of planetary systems with GAIA
}

\author{
A. Sozzetti ${ }^{1,3}$, S. Casertano ${ }^{2}$, M. G. Lattanzi ${ }^{3}$, and A. Spagna ${ }^{3}$ \\ 1 The University of Pittsburgh, Department of Physics and Astronomy, Pittsburgh, PA 15260, USA \\ 2 Space Telescope Science Institute, Baltimore, MD 21218, USA \\ 3 Osservatorio Astronomico di Torino, 10025 Pino Torinese, Italy
}

Received 8 January 2001 / Accepted 27 April 2001

\begin{abstract}
We use detailed numerical simulations and the $v$ Andromedæ planetary system as a template to evaluate the capability of the ESA Cornerstone Mission GAIA in detecting and measuring multiple planets around solartype stars in the neighborhood of the Solar System. For the outer two planets of the $v$ Andromedæ system, GAIA high-precision global astrometric measurements would provide estimates of the full set of orbital elements and masses accurate to better than 1-10\%, and would be capable of addressing the coplanarity issue by determining the true geometry of the system with uncertainties of order of a few degrees. Finally, we discuss the generalization to a variety of configurations of potential planetary systems in the solar neighborhood for which GAIA could provide accurate measurements of unique value for the science of extra-solar planets.
\end{abstract}

Key words. astrometry - stars: individual ( $v$ Andromedæ $)$ - planetary systems - space vehicles - methods: data analysis

\section{Introduction}

As of today, five normal stars in the solar neighborhood are known to harbor either two or more planets (Butler et al. 1999; Mayor et al. 2000; Udry et al. 2000; Marcy et al. 2001a) or systems composed of a planet and a brown dwarf (Udry et al. 2000; Marcy et al. 2001b; Els et al. 2001). The observational evidence of the first extra-solar planetary systems has immediately raised crucial questions regarding their formation and evolution. Are the orbits coplanar? Are the configurations dynamically stable? The quasi-coplanarity hypothesis is one of the dogmas in present planet formation theories (Lissauer 1993). For example, in the case of the three-planet system around the F8V star $v$ Andromedæ ( $v$ And hereafter), Laughlin \& Adams (1999) and Stepinski et al. (2000) have shown that orbital evolution and long-term stability of the system can be greatly affected by small variations in the three-companion orbital model. In particular, studies on the dynamical stability of the outer two companions require constraints to be placed on the maximum allowed values for the masses and on the range of allowed relative inclinations of the two orbits. Radial velocity measurements cannot determine either the inclination $i$ of the orbital plane with respect to the plane of the sky or the position angle $\Omega$ of the line of nodes in the plane of the sky: without knowledge of the full set of orbital parameters and true mass values, general conclusions on

Send offprint requests to: A. Sozzetti,

e-mail: alex@phyast.pitt.edu the architecture, orbital evolution and long-term stability of the newly discovered planetary systems may be questionable.

In this Letter, we gauge the potential of GAIA in detecting and measuring multiple planets in favorable cases, i.e. well-spaced, well-sampled orbits, and high "astrometric" $S / N$ ratios (see Sect. 3). In order to do so, we focus on the $v$ And system, which, given its characteristics, constitutes a well-suited laboratory. In this exploratory Letter, we do not address issues such as insufficient orbital sampling, resonant configurations, and astrometric signatures close to the detection limit, which clearly need to be considered in a more realistic simulation. Work on these issues is in progress and will be presented in the future.

\section{Data simulation and analysis method}

The code for the reproduction of GAIA global astrometric observations and analysis of the simulated dataset has been thoroughly described in our previous work (Lattanzi et al. 2000), where we defined detectability horizons and limits on distance for accurate orbital parameters and mass determination in the case of single Jupiter-mass planets orbiting single solar-type stars.

We have modified our simulation code to allow for the presence of multiple planets around each target star (Casertano \& Sozzetti 2000). The stellar motion is described in terms of the 5 basic astrometric parameters and of the gravitational perturbations produced by the orbiting planet(s), which are assumed to be linear (i.e., as the 
sum of independent Keplerian motions). Such simplification (no mutual interaction terms between planets) has little impact on the significance of the analysis. In fact, on the time-scale of GAIA observations (5 years), variations of the orbital parameters can be confidently considered negligible. For planet detection we employ a standard $\chi^{2}$-Test with confidence level set to $5 \%$ applied to the observation residuals (Lattanzi et al. 2000), while in the case of multiple orbits the iterative method for the solution of the non-linear systems of equations of condition is based on a slightly modified version of the LevenbergMarquardt algorithm (Casertano \& Sozzetti 1999), which ensures stability of the solution.

\section{GAIA observations of the $v$ And system}

In addressing the problem of the detection and measurement of multiple planets with GAIA, we have utilized the $v$ And planetary system as a representative test case. The results are discussed below.

\subsection{Detection}

GAIA (for a detailed review of the mission concept see Gilmore et al. 2000) surveys the sky utilizing a Hipparcos-like scanning law. Its elementary unidimensional measurement is the longitude $\psi$ of the Reference Great Circle being scanned at a given time. The single-measurement astrometric error $\sigma_{\psi}$ was fixed to $10 \mu$ as throughout all our simulations: this value applies to stars brighter than $V=12 \mathrm{mag}$. Sensitivity to planetary perturbations is driven by the astrometric error $\sigma_{\psi}$ of each measurement, which has to be compared to the magnitude of the gravitational perturbation induced on the parent star by one or more orbiting planets: the astrometric signature $\alpha$ (in arcsec) is the apparent amplitude of the orbital motion of the star around the system barycenter, defined as

$\alpha=\frac{M_{\mathrm{p}}}{M_{\star}} \frac{a_{\mathrm{p}}}{d}$,

where $M_{\mathrm{p}}, M_{\star}$ are the masses of the planet and star respectively (in solar masses), $a_{\mathrm{p}}$ the semi-major axis of the planetary orbit (in $\mathrm{AU}), d$ the distance of the system from the observer (in pc).

We simulate 200 planetary systems on the sphere using the parameters of the $v$ And system in the original paper by Butler et al. (1999), as summarized in Table 1. Two orbital elements are not determined by radial velocity measurements, namely $i$ and $\Omega$. We simulate systems with random uniform distribution in $\Omega$ and express detection probabilities as a function of the inclination of the orbital plane $\left(5^{\circ} \leq i \leq 90^{\circ}\right)^{1}$. We assume the orbits of the three planets are perfectly coplanar, i.e. $i$ and $\Omega$ are exactly the same for all the system components. Planet masses are

\footnotetext{
1 Astrometric detection probability does not depend on the apparent orientation of the orbit (Lattanzi et al. 2000).
}

Table 1. $v$ Andromedæ: stellar parameters and orbital elements utilized in the simulations. Planet masses and astrometric signatures are computed as lower limits corresponding to edge-on configurations $(\sin i=1)$.

\begin{tabular}{|c|c|c|c|}
\hline \multicolumn{2}{|c|}{ Parameters } & \multicolumn{2}{|c|}{$v$ And } \\
\hline \multirow{2}{*}{\multicolumn{2}{|c|}{$\begin{array}{l}\lambda(\operatorname{deg}) \\
\beta \text { (deg) }\end{array}$}} & \multicolumn{2}{|c|}{37.9} \\
\hline & & \multicolumn{2}{|c|}{28.9} \\
\hline \multirow{2}{*}{\multicolumn{2}{|c|}{$\begin{array}{l}\mu_{\lambda}(\operatorname{mas} / \mathrm{yr}) \\
\mu_{\beta}(\operatorname{mas} / \mathrm{yr})\end{array}$}} & \multicolumn{2}{|c|}{-242.74} \\
\hline & & \multicolumn{2}{|c|}{-266.98} \\
\hline & \multicolumn{2}{|c|}{74.25} \\
\hline \multicolumn{2}{|c|}{ Visual Magnitude } & \multicolumn{2}{|c|}{4.09} \\
\hline \multirow{2}{*}{\multicolumn{2}{|c|}{$\begin{array}{c}\text { Spectral Type } \\
d(\mathrm{pc})\end{array}$}} & \multicolumn{2}{|c|}{ F8V } \\
\hline & & & \\
\hline \multicolumn{2}{|c|}{$M_{\star}\left(M_{\odot}\right)$} & \multicolumn{2}{|c|}{1.3} \\
\hline Orbital elements & $v$ And $\mathrm{b}$ & $v$ And $\mathrm{c}$ & $v$ And $\mathrm{d}$ \\
\hline$\alpha(\mu \mathrm{as})$ & 2.40 & 100.1 & 658.4 \\
\hline$a(\mathrm{AU})$ & 0.059 & 0.83 & 2.50 \\
\hline$P(\mathrm{~d})$ & 4.6 & 241.2 & 1266.6 \\
\hline$e$ & 0.034 & 0.18 & 0.41 \\
\hline$\tau(\mathrm{JD})$ & 24500088.6 & 2450154.9 & 2451308.7 \\
\hline$\omega(\mathrm{deg})$ & 83.0 & 243.0 & 247.7 \\
\hline$M_{\mathrm{p}} \sin i\left(M_{\mathrm{J}}\right)$ & 0.71 & 2.11 & 4.61 \\
\hline
\end{tabular}

scaled with the co-secant of the inclination angle. During the 5-year mission lifetime, the system is observed for a total of 70 times $^{2}$.

We find that, given the favorable values of the "astrometric" signal-to-noise ratio $S / N=\alpha / \sigma_{\psi}$, the presence of both the outer and middle planet can be easily detected (at the 95\% confidence level) by GAIA (in the residuals to a single-star model and to a model containing the outer companion, respectively), regardless of the inclination of the orbital plane. In particular, the system configuration is such (orbits with well sampled periods, high $S / N$, no resonances) that detectability of the middle planet is not affected by the presence of the outermost companion. On the other hand, the inner planet, $v$ And $\mathrm{b}$, cannot be detected, unless $i \leq 10^{\circ}$ : as a matter of fact, its orbital period, shorter than the average observing period, is poorly sampled by GAIA, and most importantly the magnitude of the astrometric signature is such that, for $i \geq 10^{\circ}, S / N \leq 1$ and detection through the $\chi^{2}$-Test fails.

\subsection{Orbit reconstruction}

We focus here on GAIA's ability to make accurate measurements of the orbital elements and masses for the outer two planets of the system, which produce the greatest astrometric perturbations. Specifically, we have simulated systems containing the middle or the outer planet only (utilizing a single-planet solution for the orbital fits), and the complete configuration with the three planets, fitting to the observation residuals $a$ ) a two-planet model,

\footnotetext{
2 The GAIA scanning law is such that the number of basic observations is function of the ecliptic latitude $\beta$.
} 

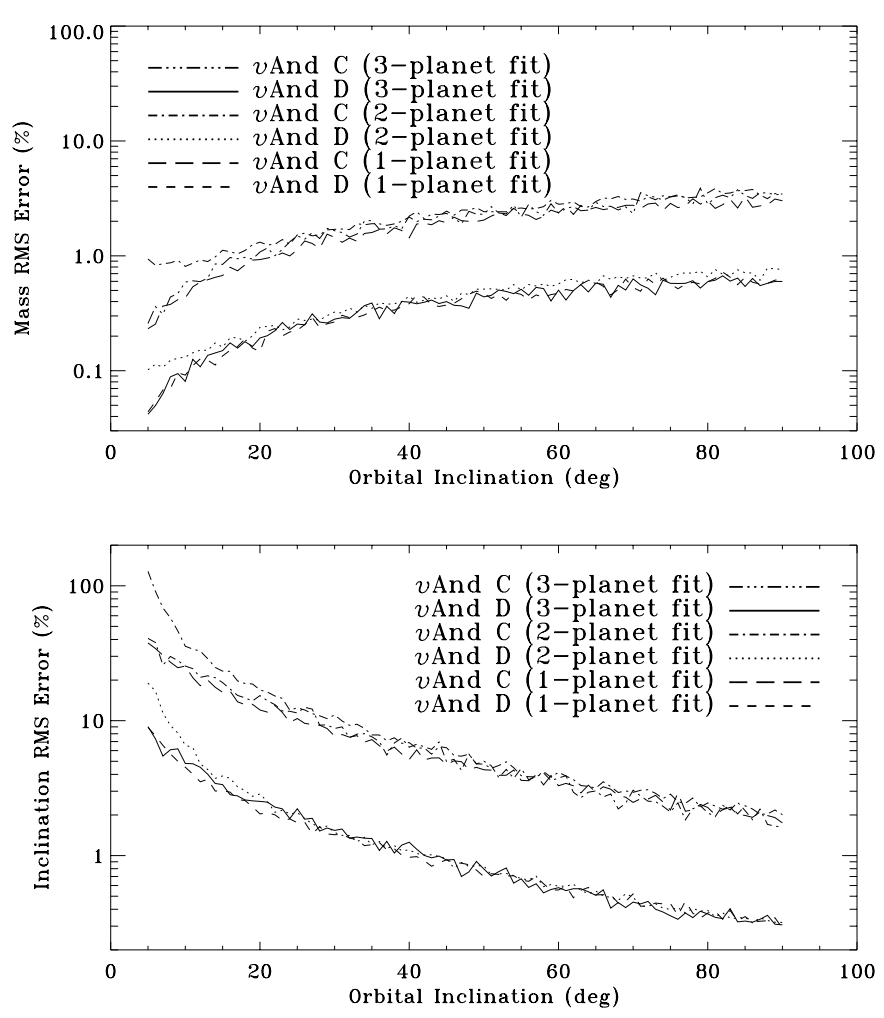

Fig. 1. rms errors for $M_{\mathrm{p}}$ and $i$, in the case of the outer two planets of the $v$ And system, expressed as fraction (\%) of the true values of the parameters: for $v$ And d, mass can be measured to $0.1-1 \%$ accuracy and inclination to better than $1 \%$ (for $i \geq 30^{\circ}$ ); for $v$ And c, mass is accurate to $1-3 \%$ and inclination to better than $10 \%$ (for $i \geq 30^{\circ}$ ).

disregarding the presence of the gravitational perturbation induced by the inner planet, or $b$ ) the complete three-planet model (for details on the fitting procedure, see Casertano \& Sozzetti 1999 or Sozzetti et al. 2001).

In Fig. 1 we have summarized the results for two of the most important parameters of the system, inclination $i$ (directly measured) and planet mass (computed once a reliable estimate for the mass of the parent star is provided), for $v$ And $\mathrm{c}$ and $v$ And d. Owing to the difference in astrometric signature (see Table 1), the outermost planet is measured more accurately than the inner planet (accuracy is almost an order of magnitude higher $)^{3}$. As the inclination $i$ decreases, towards a face-on configuration, the true mass inferred for each planet from the radial velocity data increases, therefore the astrometric signature is larger and provides a more accurate measurement of the planet's mass. At the same time, the observations are less sensitive to the inclination itself, and its fractional measurement error increases. It should also be noted that, if the system is very close to face-on, the accuracy of the inclination measurement will be substantially increased by combining radial velocity and astrometric data.

Finally, we emphasize the fact that, similarly to what happened for detection, the orbital arrangement of the

${ }^{3}$ Note that the multiple-planet fit provides very accurate estimates for all of the orbital elements of the outer two planets. $v$ And system is such that it does not affect our ability to reconstruct each planet's orbit. As shown in Fig. 1, the two outer planets can have orbital elements and masses measured almost as well as if each of them was the only orbiting companion. For a two-planet orbital fit to a complete three-planet simulation, the quality of orbit reconstruction degrades only for $i \leq 10^{\circ}$, when the signal from $v$ And $\mathrm{b}$ becomes greater than the single-measurement error (we recall $\sigma_{\psi}=10 \mu \mathrm{as}$ ), and thus detectable in the observation residuals.

\subsection{Coplanarity analysis}

As discussed in the Introduction, the unexpected orbital configuration of the $v$ And system has motivated detailed theoretical studies on its dynamical evolution and longterm stability. For example, neglecting to first order the effects of the innermost planet on the overall stability of the system, Stepinski et al. (2000) come to the conclusion that dynamical stability requires the orbital inclination of the outer two companions to be greater than $i \sim 13^{\circ}$, otherwise the two objects would be too massive and gravitational interactions would disrupt the system. Furthermore, the system cannot be stable in the long term if relative inclinations are greater than $55^{\circ}, 35^{\circ}$, and $10^{\circ}$, for $i \sim 64^{\circ}$, $i \sim 30^{\circ}$, and $i \sim 15^{\circ}$, respectively: then, the more massive the planets, the closer to coplanarity their orbits have to be, for the system not to be destabilized on a short timescale.

Figure 2 shows the estimated accuracy with which GAIA would measure the coplanarity of the orbits of $v$ And $\mathrm{c}$ and $v$ And $\mathrm{d}$ (as shown in Sect. 3.1, unless its orbit is almost face-on, $v$ And $\mathrm{b}$ is not even detectable). Fitting a three-planet model to the simulated observations, we estimate differences between position angles of the lines of nodes $\left(\Delta \Omega_{\mathrm{CD}}\right) \leq 10^{\circ}$ and differences between inclinations of the orbital planes $\left(\Delta i_{\mathrm{CD}}\right) \leq 3^{\circ}$, for $i>20^{\circ}$. Thus, quasi-coplanarity can be reliably established. As $i$ decreases, the uncertainty on $\Delta \Omega_{\mathrm{CD}}$ grows, because of the increasing difficulty in the correct identification of the position angle $\Omega$, which becomes undefined at $i=0^{\circ}$. Simulations of non-coplanar systems with $\Delta \Omega_{\mathrm{CD}} \neq 0^{\circ}$ and/or $\Delta i_{\mathrm{CD}} \neq 0^{\circ}$, yielded similar results. Our findings indicate that GAIA high-precision measurements would be instrumental in verifying the system stability, except for quasi-face on orbits: however, the inclination of $v$ And $\mathrm{d}$ is probably greater than $10^{\circ}-15^{\circ}$, otherwise the astrometry of Hipparcos would have revealed its presence (Mazeh et al. 1999).

\section{Discussion and conclusions}

The ability to accurately measure orbital parameters and masses for a potentially large number of planetary systems with massive planets is the key element of the GAIA contribution to the science of extra-solar planets.

Utilizing the $v$ And three-planet system as a template, we have shown how, in case of planetary systems with planets producing favorable astrometric signatures $(S / N \geq 10)$ and with well-sampled orbital periods 

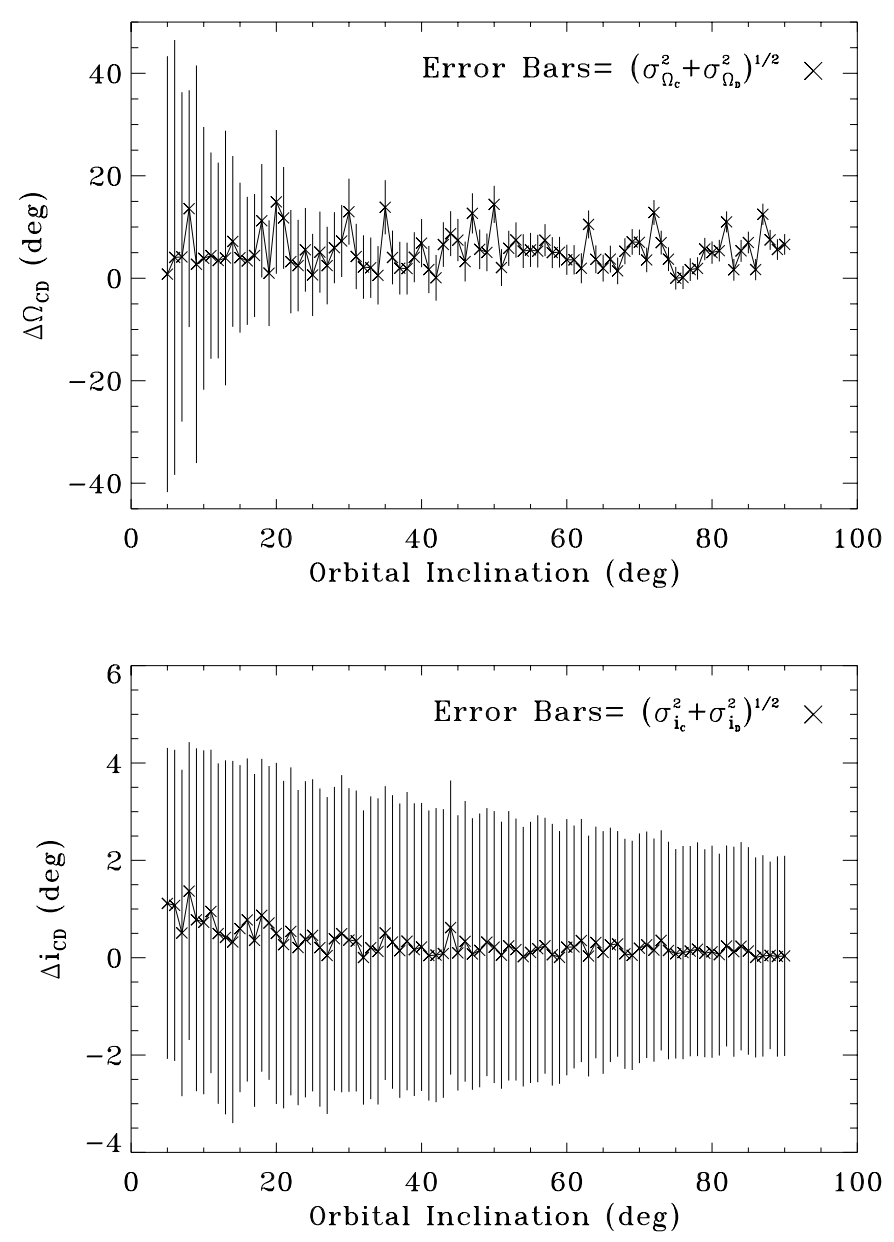

Fig. 2. Coplanarity analysis for the $v$ And system: for $i>20^{\circ}$, the orbits of the two outer planets can be confidently identified as quasi-coplanar, with (absolute) uncertainties of order of a few degrees (errors are computed using the formal expressions from the covariance matrix of the multiple-planet fit).

(shorter than the 5-year mission lifetime), the parameters and masses of each companion can be measured almost as well as if it was the only planet.

We have made coplanarity tests, and found that GAIA would be able to determine whether orbits are coplanar or not with uncertainties of a few degrees, thus providing theory with the observational evidence needed to address the long-term stability issue for the system.

Lattanzi et al. (2000) derive a 60-pc distance horizon for $30 \%$ accuracy measurements of orbital elements and masses in the case of single Jupiter-mass planets orbiting solar-type stars with periods ranging between 0.5 and 11.8 years (the true period of Jupiter).

We can generalize those findings, in an early attempt to identify the variety of possible configurations of planetary systems detectable and measurable with GAIA. The results reported in this Letter allow us to say that the 60-pc limit on distance holds for detection and measurement accurate to $30 \%$, or better, of planetary systems composed of planets with well-sampled periods $(P \leq$ $5 \mathrm{yr}$ ), and with the smaller component producing an astrometric $S / N \geq 2$. Accurate coplanarity tests will be possible for systems producing $S / N \geq 10$.

The frequency of multiple-planet systems, and their preferred orbital spacing and geometry are not currently known. Based on star counts in the vicinity of the Sun extrapolated from modern models of stellar population synthesis, constrained to bright magnitudes $(V<13 \mathrm{mag})$ and solar spectral types (earlier than K5), we should expect $\sim 13000$ stars to 60 pc (Lattanzi et al. 1999). GAIA, in its high-precision astrometric survey of the solar neighborhood, will observe each of them, searching for planetary systems composed of massive planets in a wide range of possible orbits, making accurate measurements of their orbital elements and masses, and establishing quasicoplanarity (or non-coplanarity) for detected systems with favorable configurations. The size of the stellar sample is such to ensure that the results obtained by GAIA would constitute a fundamental complement to those which will come from other planet-search programmes, in order to build the necessary statistics for deeper theoretical understanding of planetary formation processes.

\section{References}

Butler, R. P., Marcy, G. W., Fischer, D. A., et al. 1999, ApJ, 526,916

Casertano, S., \& Sozzetti, A. 1999, PASPC, 194, 171

Casertano, S., \& Sozzetti, A. 2000, JPL Technical Report (http://icarus.stsci.edu/ stefano/ planet_simulations.html)

Els, S. G., Sterzik, M. F., Marchis, F., et al. 2001, A\&A, submitted

Gilmore, G. F., de Boer, K. S., Favata, F., et al. 2000, in Proc. SPIE 4013, UV, Optical, and IR Space Telescopes and Instruments, ed. J. B. Breckinridge, \& P. Jacobsen, 453

Lattanzi, M. G., Sozzetti, A., \& Spagna, A. 1999, in From Extra-solar Planets to Cosmology: The VLT Opening Symposium, ed. J. Bergeron, \& A. Renzini (Berlin: Springer-Verlag), 479

Lattanzi, M. G., Spagna, A., Sozzetti, A., Casertano, S., et al. 2000, MNRAS, 317, 211

Laughlin, G., \& Adams, F. C. 1999, ApJ, 526, 881

Lissauer, J. J. 1993, ARA\&A, 31, 129

Marcy, G. W., Butler, R. P., Vogt, S. S., et al. 2001, ApJ, submitted

Marcy, G. W., Butler, R. P., Fischer, D., et al. 2001, ApJ, submitted

Mayor, M., Naef, D., Pepe, F., et al. 2000, in Planetary Systems in the Universe: Observation, Formation and Evolution, IAU Symp. 202, ed. A. Penny, P. Artymowicz, A. M. Lagrange, \& S. Russell, in press

Mazeh, T., Zucker, S., dalla Torre, A., et al. 1999, ApJ, 522, L149

Sozzetti, A., Brown, R., Casertano, S., et al. 2001, in preparation

Stepinski, T. F., Malhotra, R., \& Black, D. C. 2000, ApJ, 545, 1044

Udry, S., Mayor, M., \& Queloz, D. 2000, in Planetary Systems in the Universe: Observation, Formation and Evolution, IAU Symp. 202, ed. A. Penny, P. Artymowicz, A. M. Lagrange, \& S. Russell, in press 